\title{
Actividad inhibitoria de dihidroxifenil propenona sobre betalactamasas de Enterobacter cloacae: estudio preliminar en el desarrollo de fármacos para enfrentar la resistencia bacteriana
}

\author{
Cristina Lucía Mora, Julián Castaño, María Consuelo Jaramillo \\ Grupo de Investigación en Sustancias Bioactivas, Sede de Investigación Universitaria, \\ Universidad de Antioquia, Medellín, Colombia
}

\begin{abstract}
Introducción. Los microorganismos patógenos como Enterobacter cloacae producen betalactamasas que les confieren resistencia frente a los antibióticos betalactámicos; se ha identificado, además, la actividad limitada de los inhibidores enzimáticos, de modo que la única posibilidad de enfrentar la resistencia es el diseño de nuevos fármacos y su uso racional.

Objetivo. Evaluar el efecto de la chalcona dihidroxifenil propenona sobre un aislamiento clínico de $E$. cloacae y sobre la betalactamasa aislada a partir de este microorganismo resistente como un aporte en la búsqueda de compuestos inhibidores de las betalactamasas.

Materiales y métodos. Se sintetizó la chalcona dihidroxifenil propenona y se evaluó su efecto sobre el aislamiento clínico de E. cloacae para determinar la concentración inhibitoria mínima mediante el método de microdilución en caldo y con la betalactamasa purificada mediante cromatografía de afinidad se realizaron estudios espectrofotométricos de cinética enzimática.

Resultados. La concentración inhibitoria mínima de la dihidroxifenil propenona sobre E. cloacae fue de $35 \mu \mathrm{g} / \mathrm{ml}$; el porcentaje de recuperación de la betalactamasa a partir del microorganismo fue de $31,75 \%$; en el estudio cinético se evidenció actividad inhibitoria de acuerdo con los parámetros cinéticos de $\mathrm{V}_{\max }=1,7 \times 10^{-3} \mu \mathrm{M} /$ minuto y $\mathrm{K}_{\mathrm{M}}=2330 \mu \mathrm{M}$.

Conclusión. La chalcona dihidroxifenil propenona ejerce su actividad inhibitoria por medio de la interacción con la betalactamasa y, de esta manera, protege la integridad estructural de los antibióticos betalactámicos; dicho efecto sinérgico la convierte en un compuesto promisorio en la búsqueda de alternativas para enfrentar la resistencia bacteriana.
\end{abstract}

Palabras clave: Enterobacter cloacae, ampicilina, cefalosporinasa, cromatografía de afinidad, inhibidores enzimáticos, chalconas.

doi: http://dx.doi.org/10.7705/biomedica.v34i0.1668

Inhibitory activity of dihydroxy-phenyl-propenone on betalactamase of Enterobacter cloacae: Preliminary study for drug development to overcome bacterial resistance

Introduction: Enterobacter cloacae is a pathogenic microorganism with the ability to produce betalactamase enzymes, which makes them resistant to betalactamic antibiotics. Additionally, the limited activity of enzymatic inhibitors has been identified, and, therefore, the design of new drugs and the promotion of their rational use are the only possibilities to overcome this problem.

Objective: The aim of this research was to evaluate the effect of dihydroxy-phenyl-propenone on a clinical isolate of $E$. cloacae, as well as its activity on a betalactamase isolated from this resistant microorganism in order to contribute to the search for new betalactamase inhibitors.

Materials and methods: Dihydroxy-phenyl-propenone chalcone was synthesized and evaluated on a clinical isolate of $E$. cloacae to determine the minimum inhibitory concentration by broth microdilution; once the betalactamase enzyme was purified by affinity chromatography, a spectrophotometric analysis was done to evaluate its kinetic activity.

Results: The minimum inhibitory concentration value of dihydroxy-phenyl-propenone on E. cloacae was $35 \mu \mathrm{g} / \mathrm{ml}$; the recovery percentage of the betalactamase from the microorganism was $31.75 \%$ and the kinetic parameters were $\mathrm{V}_{\max }=1.7 \times 10^{-3} \mu \mathrm{M} / \mathrm{min}$ and $\mathrm{K}_{\mathrm{M}}=2330 \mu \mathrm{M}$, which show an important inhibitory activity.

\section{Contribución de los autores:}

Cristina Mora Arango: diseño de la investigación, realización de actividades experimentales y redacción del manuscrito.

Julián Castaño Zuluaga: participación en las actividades experimentales, recopilación de datos.

Consuelo Jaramillo Flórez: diseño y coordinación de la investigación, revisión del manuscrito.

Todos los autores participaron en el análisis de los resultados obtenidos. 
Conclusion: Dihydroxy-phenyl-propenone has shown inhibitory activity on betalactamase enzymes and the ability to protect the chemical integrity of betalactamic antibiotics; this synergistic effect turns it into a promising compound in the search for new alternatives to overcome bacterial resistance.

Key words: Enterobacter cloacae, ampicillin, cefalosporinase; chromatography, affinity; enzyme inhibitors, chalcones.

doi: http://dx.doi.org/10.7705/biomedica.v34i0.1668

La resistencia bacteriana se considera actualmente como un problema de salud pública de dimensiones globales. Este fenómeno se ha venido documentando desde 1940 con la introducción de la penicilina en la práctica clínica; desde entonces, los fracasos terapéuticos han sido numerosos e, infortunadamente, los intentos para controlar 0 disminuir su progreso han sido insuficientes (1-3). Por estas razones se requieren medidas urgentes que aborden el problema de forma interdisciplinaria, considerando aspectos fundamentales para detener el avance de los microorganismos resistentes tales como la ecología, la evolución de los microorganismos y la restauración de la sensibilidad a los antibióticos de uso clínico (4).

La aparición de resistencia bacteriana demuestra una vez más que en su proceso evolutivo los microorganismos han desarrollado mecanismos eficaces para evadir los blancos de acción de los antibióticos, como es el caso de las bacterias productoras de betalactamasas, enzimas con la capacidad de destruir la integridad estructural de los antibióticos derivados de la penicilina (5-11).

Existen microorganismos patógenos, como $E$. cloacae, con la capacidad de producir por vía cromosómica o constitutiva betalactamasas de clase $\mathrm{C}$ (cefalosporinasas) y, por esta razón, han desarrollado resistencia frente a los fármacos betalactámicos $(12,13)$.

Varios estudios recientes realizados en Colombia han encontrado altas tasas de resistencia para este microorganismo. En el 2009, el Grupo para el Control de la Resistencia Bacteriana de Bogotá (GREBO) presentó los porcentajes de resistencia en las unidades de cuidados intensivos de una red de hospitales en Colombia y en el caso de fármacos formulados en combinación (antibiótico betalactámico con inhibidor de betalactamasas),

\footnotetext{
Correspondencia:

Cristina Lucía Mora, Sede de Investigación Universitaria, Universidad de Antioquia, Carrera $53 \mathrm{~N}^{\circ}$ 61-30, laboratorio 229, Medellín, Colombia

Tel: (574) 219 6591; fax: (574) 2196590

cristinamora@farmacia.udea.edu.co,crisqf2@gmail.com

Recibido: 20/05/13; aceptado: 22/11/13
}

como la ampicilina y el sulbactam, la resistencia superaba el $50 \%$ (14). Asimismo, los estudios realizados en Medellín y el Valle de Aburrá por parte del Grupo para el Estudio de la Resistencia a Antibióticos en Medellín (GERMEN) entre los años 2010 y 2012 revelaron porcentajes de resistencia frente a la combinación de piperacilina y tazobactam superiores a $40 \%$ en los servicios de las unidades de cuidados intensivos y otras salas de las instituciones hospitalarias que hacen parte de la red (15).

A pesar de que los compuestos inhibidores de enzimas, como el ácido clavulánico y las sulfonas del ácido penicilánico (sulbactam y tazobactam), han ayudado a controlar la resistencia, se ha visto que su actividad es relativamente limitada contra las betalactamasas de clase $\mathrm{C}$ y contra algunas enzimas de clase A y, además, se ha reportado que estos compuestos, al presentar el mismo núcleo químico de los fármacos betalactámicos, pueden inducir la expresión de betalactamasas en microorganismos resistentes (16-22).

Frente a este panorama, la única posibilidad de detener el avance de los microorganismos resistentes es el diseño de nuevos fármacos de origen natural y sintético con diferente núcleo químico que puedan dar lugar a combinaciones efectivas de antibiótico e inhibidor, así como la promoción de su uso racional como una alternativa de valor que ayudaría a romper el círculo de la resistencia $(19,23,24)$.

En este sentido, una de las principales fuentes de compuestos con gran diversidad química y de importancia terapéutica en el tratamiento de enfermedades infecciosas la constituyen los productos naturales, en particular los compuestos conocidos como chalconas, que son flavonoides de cadena abierta con actividad antibacteriana ampliamente documentada (25-30).

Estudios recientes realizados con una serie de chalconas sintetizadas en nuestro laboratorio han demostrado actividad inhibitoria sobre la betalactamasa (31-33); por esta razón, el propósito del presente trabajo fue comparar el efecto inhibitorio de la chalcona dihidroxifenil propenona sobre un aislamiento clínico de E. cloacae y sobre la 
betalactamasa inducida y aislada a partir de este microorganismo resistente como un aporte en la búsqueda de compuestos inhibidores de la betalactamasa que puedan ser útiles para enfrentar el fenómeno de la resistencia bacteriana.

\section{Materiales y métodos}

\section{Síntesis de la dihidroxifenil propenona}

La chalcona dihidroxifenil propenona, conocida por su nombre químico como 1-(4-metil-fenil)-3-(2,3dihidroxifenil)-2-propenona, fue preparada en el laboratorio mediante la condensación de ClaissenSchmidt a partir de 1-(4-metilfenil)etanona (7,46 mmol) en medio alcalino: $\mathrm{NaOH}(0,29 \mathrm{~g}, 7,33$ $\mathrm{mmol}$ ) disuelto en agua y etanol con la adición de 2,3-dimetoxibenzaldehido (7,46 mmol). La reacción permaneció en agitación durante una hora a $25^{\circ} \mathrm{C}$ y luego se sometió a enfriamiento a $4{ }^{\circ} \mathrm{C}$ durante 12 horas $(34,35)$. Para efectuar la desmetilación se disolvió el compuesto en dimetilformamida (2 $\mathrm{ml}$ ) y yodociclohexano $(10,0 \mathrm{mmol})$ en un sistema de reacción en atmósfera inerte y en reflujo por 14 horas $(36,37)$. El producto de la reacción fue un sólido viscoso que se purificó mediante cromatografía de columna. La elucidación estructural se realizó mediante estudios de resonancia nuclear $(\mathrm{RN})$ y espectroscopia de infrarrojo. $\mathrm{RN}^{1} \mathrm{H}$ : $\left(\mathrm{CDCl}_{3}\right.$, TMS como referencia): 2,3 ppm (s, 3H), 3,7 ppm (s, 6H), 7,4 ppm (d, 1H), 7,9 ppm (d, 1H), 6,7-6,8 ppm (dd, 2H). IR: $\lambda_{\max } 3400 \mathrm{~cm}-1(\mathrm{OH}) 1177$ cm-1 (CH3), $1659 \mathrm{~cm}-1$ ( $\mathrm{C}=\mathrm{O}), 1600,1471 \mathrm{~cm}-1$ $(\mathrm{C}=\mathrm{C}$ aromático).

\section{Estudio microbiológico}

Para la determinación de la concentración inhibitoria mínima del compuesto dihidroxifenil propenona se aplicó el procedimiento de microdilución en caldo recomendado por el Clinical and Laboratory Standars Institute (CLSI) (38), con algunas modificaciones como se describe a continuación.

Aislamiento clínico de E. cloacae y preparación del inóculo bacteriano. El aislamiento clínico de $E$. cloacae fue donado por el Hospital Universitario San Vicente de Paúl con la aprobación del Comité de Ética y previo consentimiento informado del paciente, y se mantuvo por replicación en medios de cultivo e incubación a una temperatura de $37^{\circ} \mathrm{C}$. Se preparó la suspensión bacteriana en caldo $\mathrm{BHI}$ (brain-heart infusion) y se incubó durante 4 horas a $35^{\circ} \mathrm{C}$; posteriormente se aseguró una población de $5 \times 10^{5} \mathrm{UFC} / \mathrm{ml}$ en cada uno de los ensayos realizados mediante seguimiento espectrofotométrico a $625 \mathrm{~nm}$ (38).
Evaluación de la actividad inhibitoria del compuesto. La actividad inhibitoria de la chalcona dihidroxifenil propenona $(0,3-141 \mu \mathrm{g} / \mathrm{ml})$ y del tazobactam $(0,3-$ $141 \mu \mathrm{g} / \mathrm{ml}$ ) se evaluó en combinación con ampicilina a una concentración constante de $512 \mu \mathrm{g} / \mathrm{ml}$ y la determinación de la viabilidad celular posterior al tiempo de incubación de 24 horas se realizó con el reactivo TTC (2,3,5-triphenyl tetrazolium chloride), el cual se reduce por la actividad mitocondrial de las células vivas a trifenilformazán y genera un compuesto de color rojo insoluble en agua (39-42). Las lecturas espectrofotométricas posteriores a la incubación se realizaron a una longitud de onda de $530 \mathrm{~nm}$ (43).

Obtención de la enzima betalactamasa. La enzima betalactamasa clase $\mathrm{C}$, que fue el blanco molecular en los estudios de cinética enzimática, se obtuvo en el laboratorio a partir del aislamiento clínico de E. cloacae mediante los procesos de inducción, extracción y purificación.

Inducción. Se inició con un cultivo de 24 horas del aislamiento clínico de E. cloacae, el cual se ajustó a una población de microorganismo equivalente a 0,5 en la escala de McFarland, y se incubó a $37^{\circ} \mathrm{C}$ durante dos horas. A continuación se adicionó penicilina G como inductor de la producción de enzima en tres concentraciones diferentes, 0,5 ; 1,$0 ; 2,0 \mathrm{mg} / \mathrm{ml}$, y se incubó durante cinco horas con agitación continua con el fin de estandarizar la concentración efectiva para la inducción $(44,45)$. Para lograr este propósito se determinó el porcentaje de hidrólisis del nitrocefín de cada uno de los extractos crudos de betalactamasa durante diferentes tiempos de incubación, los ensayos espectrofotométricos se realizaron haciendo el seguimiento a la hidrólisis del nitrocefín $(100 \mu \mathrm{M})$ a una longitud de onda de $486 \mathrm{~nm}$ durante 60 minutos y a una temperatura de $20^{\circ} \mathrm{C}$ (46).

Extracción. Los cultivos de E. cloacae previamente inducidos para la producción de betalactamasa se centrifugaron durante 30 minutos a 2.500 rpm; los agregados celulares se suspendieron en agua desionizada y se sometieron a ciclos de ultrasonido $\left(4{ }^{\circ} \mathrm{C}\right.$ durante dos minutos en cuatro intervalos de 30 segundos) para liberar la enzima del espacio periplasmático; luego se centrifugaron las muestras durante 10 minutos y el sobrenadante (extracto total de betalactamasas) se almacenó a $-20{ }^{\circ} \mathrm{C} \mathrm{y}$ antes del estudio de cinética enzimática (45), se realizó la cuantificación de proteínas mediante el método BCA utilizando ácido bicincónico (Pierce BCA protein Assay $\mathrm{Kit}^{\mathrm{TM}}$ ) (Thermo Scientific, 
Meridian, USA). Los extractos proteicos obtenidos se liofilizaron antes de su purificación en un equipo Eyela, PFR-1000 ${ }^{\text {TM }}$ (Tokio, Japón).

Purificación. La purificación de la enzima betalactamasa se realizó mediante cromatografía de afinidad, utilizando afigel (Biorad®) modificado con ácido aminocefalosporánico (7-ACA: 7aminocephalosporanic acid) mediante la adición de EDAC (1-ethyl-3-(3-dimethylaminopropyl) carbodiimide), reactivo que permite el acoplamiento de compuestos que contienen ácidos carboxílicos y aminas primarias $(47,48)$. La elución con tampón de acetato $(\mathrm{pH} 4,4)$ seguido por tampón de fosfato $0,1 \mathrm{M}(\mathrm{pH} \mathrm{6,8)}$ permitió la obtención de fracciones purificadas de betalactamasa, las cuales se evaluaron para confirmar su actividad catalítica utilizando nitrocefín como sustrato.

Estudio cinético de la enzima $\beta$-lactamasa. La actividad catalítica y la determinación de los parámetros cinéticos $\left(\mathrm{K}_{\mathrm{M}}\right.$ y $\left.\mathrm{V}_{\max }\right)$ de la enzima betalactamasa obtenida en el laboratorio mediante cromatografía de afinidad se determinaron mediante ensayos espectrofotométricos utilizando ampicilina como sustrato en el rango de concentraciones entre 5,5-5.600 $\mu \mathrm{M}$ a una longitud de onda de $235 \mathrm{~nm}$ $(49,50)$. Los ensayos se llevaron a cabo en medio de tampón de fosfato de $0,1 \mathrm{M}(\mathrm{pH} 7,3)$ a $37^{\circ} \mathrm{C}$ y los cambios en la absorbancia se monitorizaron en un tiempo de reacción de cuatro horas con lecturas cada 10 minutos en un espectrofotómetro con lector de platos de 96 pozos (Biotek Powerwave X2 ${ }^{\text {TM }}$ ) y termostato equipado con control de temperatura. La tasa de hidrólisis y las constantes cinéticas se calcularon utilizando el software GraphPad Prism 5.0 (San Diego, California, USA).

Inhibición de betalactamasa y su cuantificación. Los estudios de inhibición sobre el blanco molecular se iniciaron con la incubación de la enzima betalactamasa purificada $(7,2 \mu \mathrm{M})$ con el inhibidor correspondiente (tazobactam como inhibidor de referencia y la dihidroxifenil propenona como inhibidor de prueba) en una concentración respectiva de 720 $\mu \mathrm{M}$ antes del seguimiento espectrofotométrico con ampicilina; cada uno de los ensayos se realizó por triplicado, y la disminución de la actividad catalítica de la enzima, debida al efecto inhibitorio, se comprobó al comparar con el control no inhibido de acuerdo con las condiciones experimentales mencionadas anteriormente en el estudio cinético (51-53).

\section{Resultados}

Al realizar los ensayos microbiológicos sobre el aislamiento clínico de E. cloacae utilizando una concentración constante de antibiótico y un rango de concentraciones de los inhibidores (tazobactam como fármaco de referencia y la chalcona dihidroxifenil propenona como compuesto de prueba), se observaron los efectos sinérgicos de ambos compuestos (cuadro 1).

Los resultados obtenidos de la concentración inhibitoria mínima indican que una concentración de $35 \mu \mathrm{g} / \mathrm{ml}$ de chalcona dihidroxifenil propenona (figura 1) combinada con una de $512 \mu \mathrm{g} / \mathrm{ml}$ de ampicilina logra la inhibición del crecimiento de la población de E. cloacae, lo cual puede estar relacionado con la interacción del compuesto con la betalactamasa, tal como ocurre en el caso del tazobactam, que logra inhibir la enzima de modo que el antibiótico, en este caso la ampicilina, queda protegido de la acción hidrolítica y, de esta manera, puede ejercer su efecto antimicrobiano, impidiendo la síntesis de peptidoglucano de la pared celular del microorganismo $(54,55)$.

El proceso de obtención de la betalactamasa se inició con el aislamiento clínico de E. cloacae, el cual fue sometido a un periodo de crecimiento en presencia de tres concentraciones de penicilina $\mathrm{G}$ como antibiótico inductor $(0,5,1,0$ y $2,0 \mathrm{mg} / \mathrm{ml})$; posteriormente se obtuvieron los extractos crudos de betalactamasa correspondientes y con ellos se realizaron estudios espectrofotométricos con el fin de seleccionar la concentración adecuada de inductor; para lograr este propósito se determinó

Cuadro 1. Concentración inhibitoria mínima de la chalcona dihidroxifenil propenona y tazobactam sobre el aislamiento clínico de Enterobacter cloacae

\begin{tabular}{lc}
\hline CIM $(\mu \mathrm{g} / \mathrm{ml})$ \\
\hline & $\begin{array}{c}\text { Aislamiento clínico de } \\
\text { Enterobacter cloacae } \\
\text { productor de betalactamasa }\end{array}$ \\
\hline Ampicilina-tazobactam & $512 / 0,3$ \\
Ampicilina-dihidroxifenil propenona & $512 / 35$ \\
\hline
\end{tabular}

CIM: concentración inhibitoria mínima<smiles>Cc1ccc(C(=O)/C=C/c2cccc(O)c2O)cc1</smiles>

Figura 1. Estructura química de la chalcona ((2E)-3-(2,3dihidroxifenil)-1-(4-etilfenil) prop-2-en-1-ona) obtenida por procedimientos de síntesis en el laboratorio 
el porcentaje de hidrólisis del nitrocefín de cada uno de los extractos crudos de betalactamasa durante los diferentes tiempos de incubación. El porcentaje de hidrólisis del nitrocefín fue un indicativo de la producción de la enzima por parte del microorganismo.

Los resultados obtenidos con los extractos proteicos provenientes del aislamiento clínico de $E$. cloacae (cepa identificada como superproductora de betalactamasa) se presentan en la figura 2 .

De acuerdo con los resultados obtenidos, las diferencias significativas entre las concentraciones evaluadas se presentaron durante los primeros 15 minutos de reacción; pasados 30 minutos se presentó más de $95 \%$ de hidrólisis con las tres concentraciones de inductor evaluadas, de modo que la concentración de inductor que favoreció una mayor tasa de hidrólisis fue la de $0,5 \mathrm{mg} / \mathrm{ml}$ de penicilina $\mathrm{G}$, debido a que en esta concentración se presentó una mayor actividad de la enzima betalactamasa en las fases iniciales de reacción.

Antes de la purificación de la betalactamasa, los extractos proteicos obtenidos se liofilizaron con el fin de asegurar la funcionalidad de la enzima y se obtuvo un total de $173,5 \mathrm{mg}$ de proteína, cuantificada mediante el método BCA.

Con el fin de aislar y purificar la betalactamasa, se aplicó el procedimiento de cromatografía de afinidad y como producto de la separación cromatográfica de $60 \mathrm{mg}$ de proteína total liofilizada, se obtuvieron 32 fracciones, en cada una de las cuales se verificó la actividad de la betalactamasa
Tiempo 0

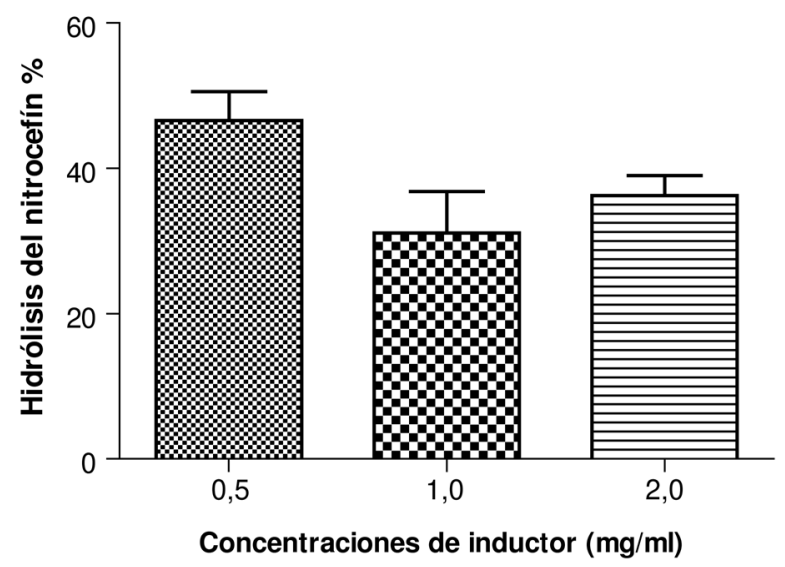

Tiempo 15

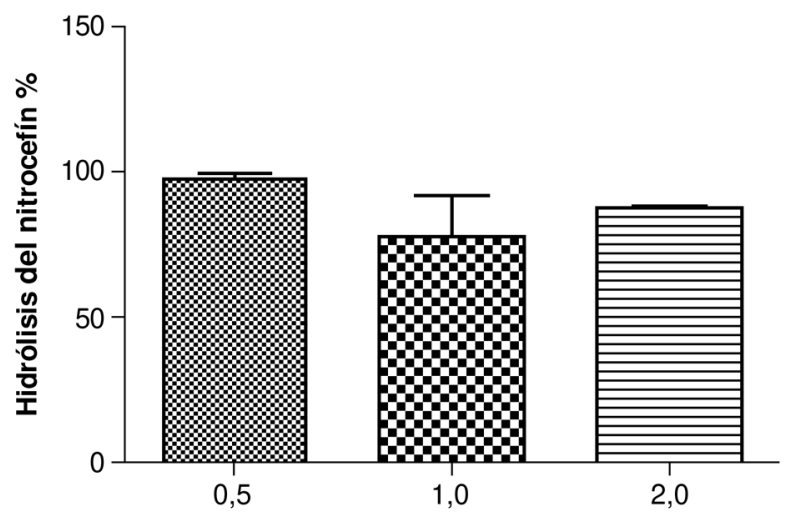

Concentraciones de inductor $(\mathrm{mg} / \mathrm{ml})$
Tiempo 5

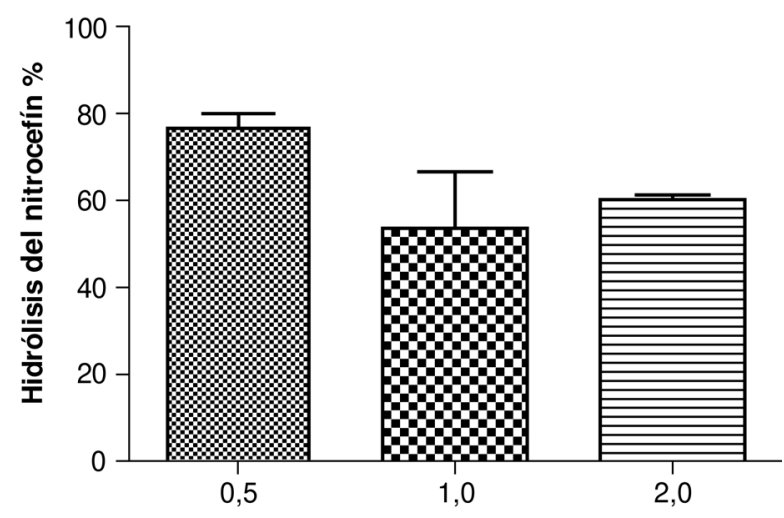

Concentraciones de inductor $(\mathrm{mg} / \mathrm{ml})$

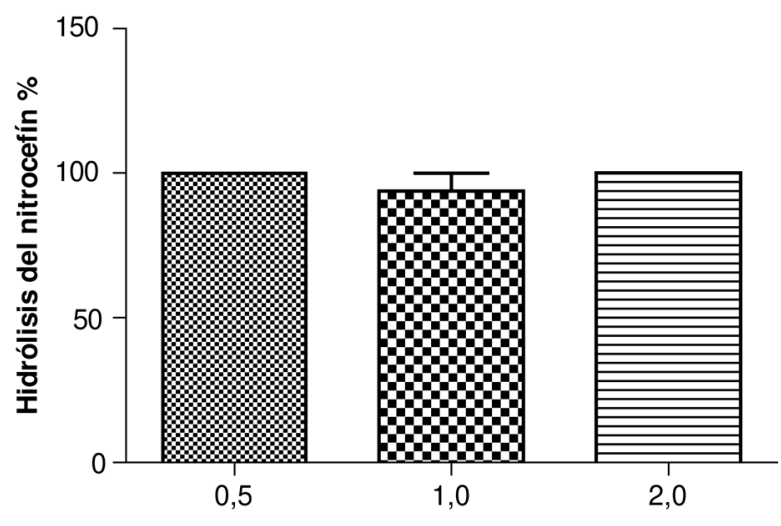

Concentraciones de inductor $(\mathrm{mg} / \mathrm{ml})$

Figura 2. Efecto de las concentraciones de penicilina G (inductor) sobre la hidrólisis del nitrocefín como medida de la actividad de la betalactamasa en los extractos proteicos crudos 
Cuadro 2. Parámetros cinéticos de la betalactamasa aislada de Enterobacter cloacae y actividad inhibitoria de la chalcona dihidroxifenil propenona y el fármaco tazobactam

\begin{tabular}{lcc}
\hline Compuesto & $\mathbf{V}_{\max }(\mu \mathrm{M} / \mathbf{m i n})$ & $\mathrm{K}_{\mathrm{M}}(\mu \mathrm{M})$ \\
\hline Ampicilina & $1,7 \times 10^{-3} \pm 3,3 \times 10^{-4}$ & $126 \pm 117$ \\
Ampicilina-tazobactam & $1,7 \times 10^{-3} \pm 3,6 \times 10^{-4}$ & $2.955 \pm 1.023$ \\
Ampicilina-dihidroxifenil & $1,7 \times 10^{-3} \pm 1,9 \times 10^{-3}$ & $2.330 \pm 607$ \\
propenona & & \\
\hline
\end{tabular}

utilizando nitrocefín como sustrato. El contenido proteico de las fracciones activas se cuantificó mediante el método BCA, obteniéndose un porcentaje de recuperación de la betalactamasa de $31,75 \%$. Los resultados de la cuantificación de proteínas en las fracciones activas se presentan en la cuadro 2.

La fracción de betalactamasa obtenida en el procedimiento de cromatografía de afinidad en la que se presentó la mayor concentración fue la fracción 3 con una concentración de 7.051,664 $\mu \mathrm{g} /$ $\mathrm{ml}$ : esta fracción se diluyó hasta una concentración de 2.820,666 $\mathrm{\mu g} / \mathrm{ml}$, de tal manera que la concentración definitiva en los estudios de cinética enzimática fue de $282,066 \mu \mathrm{g} / \mathrm{ml}$, equivalente a una concentración de 7,2 $\mu \mathrm{M}$ de betalactamasa purificada. La concentración de betalactamasa en el extracto proteico representa una cantidad de enzima de $60 \mathrm{ng}$, lo que equivale a 0,02 unidades de actividad enzimática con la capacidad de hidrolizar $0,02 \mu \mathrm{mol}$ de sustrato en un tiempo de 0,02 minutos ( 1,2 segundos); esta concentración de enzima garantiza la relación molar (1/1) de betalactamasa y ampicilina para la aplicación del estudio cinético, en el que las cantidades de sustrato se encuentran en el rango de 0,011-1,120 $\mu \mathrm{mol}$ (equivalentes a 5,5-5.600 $\mu \mathrm{M}$ ) con el fin de asegurar cantidades superiores e inferiores a $0,02 \mu \mathrm{mol}$ de ampicilina $(56,57)$.

Con el objeto de verificar la actividad catalítica de la enzima purificada mediante cromatografía de afinidad, se realizaron estudios de cinética enzimática utilizando ampicilina como sustrato y se calcularon las constantes cinéticas de velocidad máxima $\left(\mathrm{V}_{\max }\right)$ y constante de afinidad $\left(\mathrm{K}_{\mathrm{M}}\right)$. Los resultados obtenidos en estos parámetros cinéticos describen el comportamiento catalítico de la enzima purificada, el cual se ajustó al modelo descrito por Michaelis y Menten, en el cual la actividad cinética de la betalactamasa obtenida se caracteriza por el aumento de la velocidad catalítica en función del aumento de las concentraciones de ampicilina hasta llegar a la etapa de saturación de la enzima

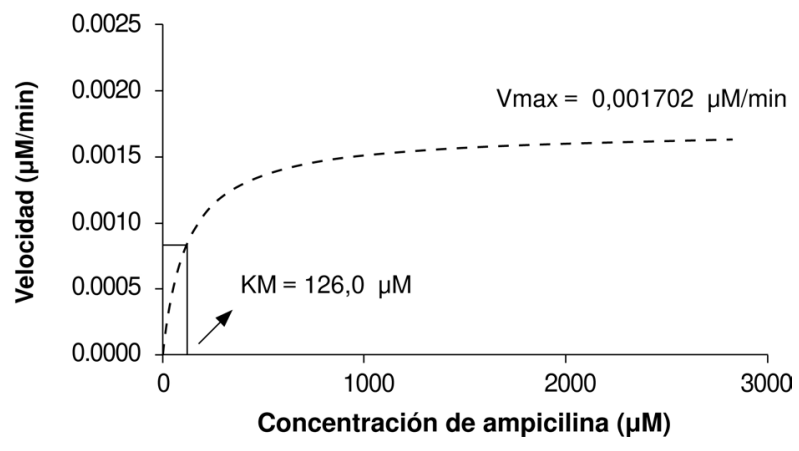

Figura 3. Gráfica de Michaelis y Menten correspondiente a la actividad catalítica de la betalactamasa purificada

en la cual se alcanza la velocidad máxima de reacción y que permanece constante ante el aumento de las concentraciones de sustrato (5860) (figura 3).

Con base en los resultados de actividad catalítica de la betalactamasa obtenida a partir del aislamiento clínico de E. cloacae con ampicilina como sustrato, se comprobó el potencial inhibitorio de la chalcona dihidroxifenil propenona evaluada a una concentración constante de $720 \mu \mathrm{M}$; al realizar los estudios de cinética enzimática en combinación con el antibiótico ampicilina, se propuso un análisis comparativo para detectar los cambios en los parámetros cinéticos debido a la presencia del fármaco tazobactam como inhibidor de referencia y la chalcona dihidroxifenil propenona como compuesto de prueba, conservando en los dos inhibidores evaluados una proporción de betalactamasa e inhibidor de 1/100 (53). Los resultados obtenidos se presentan en el cuadro 3.

La actividad catalítica de la enzima betalactamasa se vio afectada en presencia de los dos inhibidores evaluados debido a la disminución de la afinidad de la enzima por el sustrato ampicilina, lo que permitió observar el aumento de las constantes de afinidad aparentes $\left(\mathrm{K}_{\mathrm{M}}\right)$ en presencia del tazobactam y de la chalcona dihidroxifenil propenona; ambos compuestos ejercieron su actividad inhibitoria sin afectar la velocidad máxima catalítica, indicando el tipo de inhibición competitiva en la reacción.

Cuando se evaluó la enzima sin inhibir, la concentración de ampicilina necesaria para alcanzar la mitad de la velocidad máxima catalítica fue de $126 \mu \mathrm{M}$, mientras que cuando se evaluó la enzima inhibida con la chalcona dihidroxifenil propenona, se requirió una concentración de $2.330 \mu \mathrm{M}$ de ampicilina para alcanzar este parámetro; por otro lado, con el tazobactam como inhibidor de 
Cuadro 3. Resultados de la cuantificación de proteínas mediante el método BCA de las fracciones que presentaron actividad de betalactamasa obtenidas mediante cromatografía de afinidad

\begin{tabular}{cc}
\hline Fracción & Contenido de proteína $(\boldsymbol{\mu g} / \mathrm{ml}) \pm \mathrm{DE}$ \\
\hline 2 & $3.973,823 \pm 0,05186$ \\
3 & $7.051,664 \pm 0,02400$ \\
4 & $6.149,464 \pm 0,03745$ \\
6 & $841,303 \pm 0,007082$ \\
10 & $462,149 \pm 0,004634$ \\
11 & $471,502 \pm 0,02951$ \\
12 & $103,139 \pm 0,02248$ \\
\hline
\end{tabular}

referencia, la afinidad disminuyó significativamente hasta un valor de $2.955 \mu \mathrm{M}$ de ampicilina, lo cual deja en evidencia el potencial inhibitorio del compuesto sintetizado y puede interpretarse como una mayor afinidad de fijación de la enzima por los inhibidores y no por el sustrato natural representado por la ampicilina como antibiótico betalactámico, de modo que la acción de los inhibidores es evitar la formación del complejo betalactamasa-ampicilina responsable de la hidrólisis e inactivación del fármaco betalactámico.

\section{Discusión}

Los estudios cinéticos y microbiológicos realizados en nuestro laboratorio para evaluar una serie de chalconas con diferentes patrones de sustitución han demostrado el potencial de este grupo de compuestos en la restauración de la actividad de antibióticos betalactámicos frente a microorganismos resistentes productores de betalactamasa (31-33).

Los resultados promisorios nos han motivado a continuar avanzando en el estudio de la actividad inhibitoria de la chalcona dihidroxifenil propenona sobre aislamientos de microorganismos patógenos resistentes de importancia clínica por la producción de betalactamasa, como E. cloacae. El tema resulta de gran interés debido a la prevalencia en nuestro medio de infecciones hospitalarias causadas por este microorganismo asociado con tasas muy altas de resistencia; en este contexto surge como alternativa la chalcona dihidroxifenil propenona, cuyo potencial inhibitorio radica en sus características químicas y que, al no ser estructuralmente análoga de los inhibidores con núcleo betalactámico conocidos actualmente (sulbactam, tazobactam, ácido clavulánico), podría interactuar con la betalactamasa y ejercer su efecto inhibitorio, evitando así los fenómenos de resistencia cruzada a fármacos y la inducción enzimática, los cuales se consideran como la principal amenaza en el desarrollo de nuevos inhibidores enzimáticos $(61,62)$.
En el estudio microbiológico, el aislamiento clínico de E. cloacae no presentó inhibición en el crecimiento relacionada con la concentración de ampicilina evaluada y debida a la producción de betalactamasas como mecanismo de resistencia; cuando se evaluó el efecto antimicrobiano de los inhibidores por separado tampoco se presentó inhibición en el crecimiento. Por otro lado, cuando se evaluó el tazobactam como inhibidor de referencia y la chalcona dihidroxifenil propenona como compuesto de prueba en combinación con el antibiótico ampicilina, se evidenció el efecto sinérgico de la ampicilina y la dihidroxifenil propenona en las concentraciones respectivas de $512 / 35 \mu \mathrm{g} / \mathrm{ml}$. Dicha combinación logró inhibir el crecimiento del microorganismo, lo cual deja en claro que el compuesto sintetizado carece de actividad antimicrobiana y que su actividad podría estar relacionada con la interacción con la enzima betalactamasa producida por el microorganismo.

Con el fin de profundizar en la investigación y conocer los efectos del compuesto sintetizado sobre la betalactamasa como blanco molecular, se aisló y se purificó la enzima producida por el microorganismo E. cloacae y se realizó el estudio cinético correspondiente. Con este se demostró que la chalcona dihidroxifenil propenona compitió por los sitios activos de la betalactamasa y, probablemente mediante la formación de un complejo estable de betalactamasa y dihidroxifenil propenona, disminuyó la afinidad de la enzima por el sustrato ampicilina, lo cual se vio reflejado en el alto valor obtenido en el cálculo de la constante de afinidad aparente $\mathrm{K}_{\mathrm{M}}=2.330 \mu \mathrm{M}$.

Así, pues, la chalcona dihidroxifenil propenona ejerció su actividad inhibitoria por medio de la interacción con la betalactamasa y protegió la integridad estructural de los antibióticos betalactámicos, de tal manera que por su efecto sinérgico se convierte en un compuesto promisorio en la búsqueda de alternativas para enfrentar la resistencia bacteriana.

Los resultados del presente trabajo permiten evidenciar el potencial inhibitorio de la chalcona dihidroxifenil propenona y brindan la posibilidad de continuar con el estudio de la interacción entre enzima e inhibidor, el mecanismo de acción inhibitorio del compuesto y las características de permeabilidad celular y actividad citotóxica como un valioso aporte en el desarrollo de nuevos fármacos que mediante la inhibición de enzimas hidrolíticas puedan proteger la integridad estructural 
de los antibióticos betalactámicos, ampliamente conocidos en la práctica clínica por sus perfiles de seguridad y eficacia en el tratamiento de las enfermedades infecciosas.

\section{Agradecimientos}

Los autores agradecemos al Grupo de Investigación en Sustancias Bioactivas, especialmente a las jóvenes investigadoras Adriana Castaño y Natalia Gómez por su apoyo constante en las actividades experimentales y a los investigadores Diana Colorado y Óscar Lara por su colaboración en la aplicación de los métodos de cuantificación de proteínas.

\section{Conflicto de intereses}

Los autores declaramos que no existe ningún conflicto de intereses en la publicación de este trabajo de investigación.

\section{Financiación}

El presente trabajo de investigación fue financiado por la Universidad de Antioquia y el Grupo de Investigación en Sustancias Bioactivas de la Corporación de Patologías Tropicales de la Universidad de Antioquia a través del proyecto 341344 y del proyecto CODI - Estrategia de sostenibilidad GISB 2013-2014.

\section{Referencias}

1. Neu HC. The crisis in antibiotic resistance. Science. 1992;257:1064-73. http://dx.doi.org/10.1126/science.257. 5073.1064

2. Stratton CW. The clinical implications of $\beta$-lactamase. Antimicrobics Infect Dis Newsletter. 1996;15:67-71.

3. Crespo MP. La resistencia bacteriana: ¿estamos preparados para detectarla? Infectio. 2005;9:31-45

4. Baquero F, Coque TM, De la Cruz F. Ecology and evolution as targets: The need for novel Eco-Evo drugs and strategies to fight antibiotic resistance. Antimicrob Agents Chemother 2011;55:3649-60. http://dx.doi.org/10.1128/AAC.00013-11

5. Babic M, Hujer AM, Bonomo RA. What's new in antibiotic resistance? Focus on $\beta$-lactamase. Drug Resist Updat. 2006;9:142-6. http://dx.doi.org/10.1016/j.drup.2006.05.005

6. Galeni M, Lamotte-Brasseur J, Raquet X. The enigmatic catalytic mechanism of active-site serine $\beta$-lactamase. Biochem Pharmacol. 1995;49:1171-8. http://dx.doi.org/10. 1016/0006-2952(94)00502-D

7. Frère $\mathbf{J}-\mathbf{M}$. Beta-lactamases and bacterial resistance to antibiotics. Mol Microbiol.1995;16:385-95. http://dx.doi.org/ 10.1111/j.1365-2958.1995.tb02404.x

8. Bush K. Characterization of $\beta$-lactamases. Antimicrob Agents Chemother. 1989;33:259-63. http://dx.doi.org/10.1128/AAC. 33.3.259

9. Bush K, Jacoby GA, Medeiros AA. A functional classification scheme for $\beta$-lactamases and its correlation with molecular structure. Antimicrob Agents Chemother. 1995;39:1211-33. http://dx.doi.org/ 10.1128/AAC.39.6.1211

10. Livermore DM. $\beta$-Lactamase-mediated resistance and opportunities for its control. J Antimicrob Chemother. 1998; 41(Suppl.D):25-41. http://dx.doi.org/10.1093/jac/41.suppl_4.25

11. Thomson K, Moland ES. Version 2000: The new $\beta$-lactamases of Gram-negative bacteria at the dawn of the new millennium. Microbes Infect. 2000;2:1225-35. http://dx. doi.org/10.1016/S1286-4579(00)01276-4

12. Livermore DM, Maskell JP, Williams D. Detection of PSE-2 $\beta$-lactamase in Enterobacteriaceae. Antimicrob Agents Chemother. 1984;25:268-72. http://dx.doi.org/10.1128/AAC. 25.2.268

13. Marchou B, Bellido F, Charnas R, Lucain C, Pechère JC. Contribution of beta-lactamase hydrolysis and outer membrane permeability to ceftriaxone resistance in Enterobacter cloacae. Antimicrob Agents Chemother. 1987;31:1589-95. http://dx. doi.org/10.1128/AAC.31.10.1589

14. Grupo para el Control de la Resistencia Bacteriana de Bogotá (GREBO). Boletín 2009. Fecha de consulta: 16 de mayo de 2013. Disponible en: http://www.grebo.org/ BOLETiN\%202009_web.pdf.

15. Grupo para el Estudio de la Resistencia a Antibióticos en Medellín (GERMEN). Perfiles de sensibilidad a antibióticos de Enterobacter cloacae. Fecha de consulta: 16 de mayo de 2013. Disponible en: http://www.grupogermen.org/pdf/ enterobacter.pdf.

16. Coleman K. Extending the life of $\beta$-lactam antibiotics: New $\beta$-lactamase inhibitors. Drug Discov Today Ther Strateg. 2006;3:183-8. http://dx.doi.org/10.1016/j.ddstr.2006.06.010

17. Blázquez J, Baquero MR, Cantón R, Alos I, Baquero F. Characterization of a new TEM-type $\beta$-Lactamase resistant to clavulanic acid, sulbactam and tazobactam in clinical isolates of Escherichia coli. Antimicrob Agents Chemother. 1993;37:2059-63. http://dx.doi.org/ 10.1128/AAC. 37.10.2059

18. Li Q, Lee JY, Castillo R, Hixon MS, Pujol C, Doppalapudi VR, et al. NB2001, a novel antibacterial agent with broadspectrum activity and enhanced potency against $\beta$-lactamaseproducing strains. Antimicrob Agents Chemother. 2002;46: 1262-8. http://dx.doi.org/ 10.1128/AAC.46.5.1262-1268.2002

19. Arpin C, Labia R, Dubois V, Noury P, Souquet M, Quentin C. TEM-80, a novel inhibitor-resistant $\beta$-Lactamase in a clinical isolate of Enterobacter cloacae. Antimicrob Agents Chemother. 2002;46:1183-90. http://dx.doi.org/10.1128/AAC. 46.5.1183-1189.2002

20. Thomson KS, Weber DA, Sanders CC, Sanders WE Jr. $\beta$-lactamase production in members of the family Enterobacteriaceae and resistance to $\beta$-lactam-enzyme inhibitor combinations. Antimicrob Agents Chemother. 1990;34:622-7. http://dx.doi.org/ 10.1128/AAC.34.4.622

21. Yang Y, Rasmussen B, Shlaes DM. Class A $\beta$-lactamaseenzyme-inhibitor interactions and resistance. Pharmacol Ther. 1999;83:141-51. http://dx.doi.org/10.1016/S0163-7258 (99)00027-3

22. Helfand MS, Bethel CR, Hujer AM, Hujer KM, Anderson VE, Bonomo RA. Understanding resistance to betalactams and beta-lactamase inhibitors in the SHV betalactamase: Lessons from the mutagenesis of SER-130. J 
Biol Chem. 2003;278:52724-9. http://dx.doi.org/10.1074/jbc. M306059200

23. Kuck NA, Jacobus NV, Petersen PJ, Weiss WJ, Testa RT. Comparative in vitro and in vivo activities of piperacillin combined with the $\beta$-lactamase inhibitors tazobactam, clavulanic acid, and sulbactam. Antimicrob Agents Chemother. 1989;33:1964-9. http://dx.doi.org/10.1128/AAC.33.11.1964

24. Payne DJ, Cramp R, Winstanley DJ, Knowles DJ. Comparative activities of clavulanic acid, sulbactam, and tazobactam against clinically important $\beta$-lactamases. Antimicrob Agents Chemother. 1994;38:767-72. http://dx.doi. org/10.1128/AAC.38.4.767

25. Cushnie TP, Lamb AJ. Recent advances in understanding the antibacterial properties of flavonoids. Int $\mathrm{J}$ Antimicrob Agents.2011;38:99-107.http://dx.doi.org/10.1016/j.ijantimicag. 2011.02.014

26. Nowakowska Z. A review of anti-infective and antiinflammatory chalcones. Eur J Med Chem. 2007;42:125-37. http://dx.doi.org/10.1016/j.ejmech.2006.09.019

27. Pereira H, Albino E de F, Delle Monache F, Smânia Jr A. Structure-activity relationship of antibacterial chalcones. Bioorg Med Chem. 2008;16:9790-4. http://dx.doi.org/10. 1016/j.bmc.2008.09.064

28. Nowakowska Z, Kędzia B, Schroeder G. Synthesis, physicochemical properties and antimicrobial evaluation of new (E)- Chalcones. Eur J Med Chem. 2008;43:707-13. http://dx.doi.org/10.1016/j.ejmech.2007.05.006

29. Batovska D, Parushev S, Stamboliyska B, Tsvetkova I, Ninova M, Najdenski H. Examination of growth inhibitory properties of synthetic chalcones for which antibacterial activity was predicted. Eur J Med Chem. 2009;44:2211-8. http://dx.doi.org/10.1016/j.ejmech.2008.05.010

30. Liu XL, Xu YJ, Go ML. Functionalized chalcones with basic functionalities have antibacterial activity against drug sensitive Staphylococcus aureus. Eur J Med Chem. 2008;43:1681-7. http://dx.doi.org/10.1016/j.ejmech.2007.10.007

31. Jaramillo MC, Mora CL, Vélez LE, Quijano J. Kinetic and theoretical study of chalcones as inhibitors of $\beta$-lactamase enzyme. Med Chem. 2009;5:434-9. http://dx.doi.org/ 10.2174/ 157340609789117895

32. Jaramillo MC, Mora CL, Vélez LE, Arango GJ, Quijano J. Biological evaluation of aromatic compounds as $\beta$-lactamase inhibitors. Pharmacologyonline. 2005;3:650-5.

33. Mora CL, Jaramillo MC, Vélez LE, Sierra JA. Determinación de la capacidad inhibitoria de chalconas sobre Staphylococcus aureus productor de $\beta$-lactamasa. Not Quím. 2008;30:91-2.

34. Furniss BS, Tatchell AR, Hannaford AJ, Smith PW. Vogel's textbook of practical organic chemistry. Fifth Edition. London: Prentice Hall; 1989. p. 1514.

35. Calvino V, Picallo M, López-Peinado AJ, Martín-Aranda RM, Durán-Valle CJ. Ultrasound accelerated ClaisenSchmidt condensation: A green route to chalcones. Appl Surf Sci. 2006;252:6071-4. http://dx.doi.org/10.1016/j.apsusc. 2005.11.006

36. McOmie JFW, Watts ML, West DE. Demethylation of aryl methyl ethers by boron tribromide. Tetrahedron. 1968;24:228992. http://dx.doi.org/10.1016/0040-4020(68) 88130-X
37. Zuo L, Yao S, Wang W, Duan W. An efficient method for demethylation of aryl methyl ethers. Tetrahedron Lett. 2008;49:4054-6. http://dx.doi.org/10.1016/j.tetlet.2008.04.070

38. Clinical and Laboratory Standards Institute. Performance standards for antimicrobial susceptibility testing; twenty-first informational supplement. Wayne: CLSI; 2011.

39. Lampe MF, Minshew BH, Sherris JC. In vitro response of Enterobacter to ampicillin. Antimicrob Agents Chemother. 1979;16:458-62. http://dx.doi.org/10.1128/AAC.16.4.458

40. Strayer AH, Gilbert DH, Pivarnik P, Medeiros AA, Zinner SH, Dudley MN. Pharmacodynamics of piperacillin alone and in combination with tazobactam against piperacillinresistant and susceptible organisms in an in vitro model of infection. Antimicrob Agents Chemother. 1994;38:2351-6. http://dx.doi.org/10.1128/AAC.38.10.2351

41. Karlowsky JA, Weaver MK, Thornsberry C, Dowzicky MJ, Jones ME, Sahm DF. Comparison of four antimicrobial susceptibility testing methods to determine the in vitro activities of piperacillin and piperacillin-tazobactam against clinical isolates of Enterobacteriaceae and Pseudomonas aeruginosa. J Clin Microbiol. 2003;41:3339-43. http://dx.doi. org/ 10.1128/JCM.41.7.3339-3343.2003

42. Stock I, Grüger T, Wiedemann B. Natural antibiotic susceptibility of strains of the Enterobacter cloacae complex. Int J Antimicrob Agents. 2001;18:537-45. http://dx.doi.org/10. 1016/S0924-8579(01)00463-0

43. Michel T, Destandau E, Le Floch G, Lucchesi M. E, Elfakir C. Antimicrobial, antioxidant and phytochemical investigations of sea buckthorn (Hippohae rhamnoides L.) leaf, stem, root and seed. Food Chem. 2012;131 754-60. http://dx.doi.org/10.1016/j.foodchem.2011.09.029

44. Hennessey TD. Inducible $\beta$-lactamase in Enterobacter. J Gen Microbiol. 1967;49:277-85. http://dx.doi.org/10.1099/0022128749-2-277

45. Gootz TD, Sanders CC. Characterization of $\beta$-lactamase induction in Enterobacter cloacae. Antimicrob Agents Chemother. 1983;23:91-7. http://dx.doi.org/ 10.1128/AAC. 23.1.91

46. Farmer TH, Page JW, Payne DJ, Knowles DJ. Kinetic and physical studies of $\beta$-lactamase inhibition by a novel penem, BRL 42715. Biochem J. 1994;303:825-30.

47. Eriquez LA, D'Amato RF. Purification by affinity chromatography and properties of a $\beta$-lactamase isolated from Neisseria gonorrhoeae. Antimicrob Agents Chemother. 1979;15:229-34. http://dx.doi.org/ 10.1128/AAC.15.2.229

48. Minami S, Inoue M, Mitsuhashi S. Purification and properties of a cephalosporinase from Enterobacter cloacae. Antimicrob Agents Chemother. 1980;18:853-7. http://dx.doi. org/ 10.1128/AAC.18.6.853

49. Galleni M, Amicosante G, Frére JM. Sequence and comparative analysis of three Enterobacter cloacae ampC $\beta$-lactamase genes and their products. Biochem J. 1988; 225123-9.

50. Monnaie D, Virden R, Frére JM. A rapid kinetic study of the class C $\beta$-lactamase of Enterobacter cloacae 908R. FEBS J. 1992;306:108-12. http://dx.doi.org/10.1016/00145793(92)80979-Q

51. Stapleton PD, Shah S, Anderson JC, Hara Y, Jeremy $\mathbf{M T}$, Miller $\mathbf{H}$, et al. Modulation of $\beta$-lactam resistance in 
Staphylococcus aureus by catechins and gallates. Int J Antimicrob Agents. 2004;23:462-7. http://dx.doi.org/10.1016/j. ijantimicag.2003.09.027

52. Yang $Y$, Janota $K$, Tabei $K$, Huang $N$, Siegel M, Lin Yl, et al. Mechanism of inhibition of the class A $\beta$-lactamases PC1 and TEM-1 by tazobactam. J Biol Chem. 2000;275:2667482. http://dx.doi.org/ 10.1074/jbc.M002369200

53. Bonomo R, Liu J, Chen Y, Ng L, Hujer AM, Anderson VE. Inactivation of CMY-2 $\beta$-lactamase by tazobactam: Initial mass spectroscopic characterization. Biochim Biophys Acta. 2001;1547:196-205. http://dx.doi.org/10.1016/S01674838(01)00175-3

54. Vardanyan R, Hruby V. Synthesis of essential drugs. First edition. Netherlands: Elsevier B.V; 2006. p. 425-98.

55. Papp-Wallace KM, Bethel CR, Gootz TD, Shang W, Stroh J, Lau W, et al. Inactivation of a class A and a class C $\beta$-lactamase by $6 \beta$-(hydroxymethyl)penicillanic acid sulfone. Biochem Pharmacol. 2012;83:462-71. http://dx.doi. org/10.1016/j.bcp.2011.11.015

56. Galleni M, Amicosante G, Frére JM. A survey of the kinetic parameters of class C $\beta$-lactamases. Biochem J. 1988;255:123-9.

57. Tsang $\mathbf{M - W}$, Leung YC. Overexpression of the recombinant Enterobacter cloacae P99 AmpC $\beta$-lactamase and its mutants based on a $\varphi 105$ prophage system in Bacillus subtilis. Protein Expression Purif. 2007;55:75-83. http:// dx.doi.org/10.1016/j.pep.2007.06.001

58. Lejeune A, Vanhove M, Lamotte-Brasseur J, Pain RH, Frère JM, Matagne A. Quantitative analysis of the stabilization by substrate of Staphylococcus aureus PC1 $\beta$-lactamase. Chem Biol. 2001;8:831-42. http://dx.doi.org/10.1016/S10745521(01)00053-9

59. Swaren P, Maveyraud L, Guillet V, Masson J-M, Mourey L, Samam JP. Electrostatic analysis of TEM1 $\beta$-lactamase: Effect of substrate binding, steep potential gradients and consequences of site-directed mutations. Structure. 1995;3:603-13. http://dx.doi.org/10.1016/S0969-2126(01) 00194-0

60. Majiduddin FK, Materon IC, Palzkill TG. Molecular analysis of beta-lactamase structure and function. Int J Med Microbiol. 2002;292:127-37. http://dx.doi.org/10.1078/14384221-00198

61. Eumkeb G, Chukrathok S. Synergistic activity and mechanism of action of ceftazidime and apigenin combination against ceftazidime-resitant Enterobacter cloacae. Phytomedicine. 2013;20:262-9. http://dx.doi.org/10.1016/j. phymed.2012.10.008

62. Coleman K. Diazabicyclooctanes (DBOs): A potent new class of non $\beta$-lactam $\beta$-lactamase inhibitors. Curr Opin Microbiol. 2011;14:550-5. http://dx.doi.org/10.1016/j.mib. 2011.07.026 